\title{
CT-Scan Presentations of Brain Malformations in Children: About Three Cases in Regional Hospital of Ngaoundere-Cameroon
}

\author{
Neossi Guena Mathurin ${ }^{1,2 *}$, Alapha Florent' ${ }^{2}$, Doka Kamo Héléne ${ }^{3}$ \\ ${ }^{1}$ Department of Biomedical Sciences, Faculty of Science, The University of Ngaoundere, Ngaoundere, Cameroon \\ ${ }^{2}$ Medical Imaging Center of Ngaoundere Regional Hospital, Ngaoundere, Cameroon \\ ${ }^{3}$ Peadiatric Department of Ngaoundere Regional Hospital, Ngaoundere, Cameroon \\ Email: *mneossiguena@yahoo.fr
}

How to cite this paper: Mathurin, N.G., Florent, A. and Héléne, D.K. (2018) CT-Scan Presentations of Brain Malformations in Children: About Three Cases in Regional Hospital of Ngaoundere-Cameroon. Open Journal of Radiology, 8, 74-83.

https://doi.org/10.4236/ojrad.2018.82009

Received: April 21, 2018

Accepted: June 12, 2018

Published: June 19, 2018

Copyright $\odot 2018$ by authors and Scientific Research Publishing Inc. This work is licensed under the Creative Commons Attribution International License (CC BY 4.0).

http://creativecommons.org/licenses/by/4.0/

\begin{abstract}
Brain malformations are rare, difficult to diagnose and have unpredictable evolution. They are the major causes of epilepsy, psychomotor development abnormalities and other neurological disorders. The neuroimaging technique of choice for diagnosis of these malformations is magnetic resonance imaging (MRI), but unfortunately MRI is expensive, and is not available in a poor resource country like Cameroon. CT scan associated to clinical signs can help to suspect or to confirm a malformation. The authors report here three cases of malformations discovered during cranial CT scan at the regional hospital of Ngaoundéré. They are Dandy Walker malformation, Sturge Weber's disease and hemimegalencephaly. These cases contribute to the knowledge of this rare event, and emphasize the importance of CT scan on their diagnosis in the absence of MRI.
\end{abstract}

\section{Keywords}

Brain Malformations, Cerebral CT-Scan, Dandy Walker, Sturge Weber Diseases, Hemimegalencephaly, Poor Resource Country

\section{Introduction}

Brain malformations (BMs) are congenital damage or abnormal development of the brain. These BMs are due to a halt or a modification of brain development during intrauterine life, regardless of the etiology. Sometimes it's a genetic problem. In other cases, exposure to certain medicines, infections, or radiation during pregnancy interferes with brain development; something damages the developing nervous system or causes it to develop abnormally [1]. Central nervous 
system malformations are rare, difficult to diagnose and have unpredictable evolution. The hospital prevalence of $0.52 \%$ in neuropediatric consultations was reported by Moifo et al. in Cameroon [2]. In other series they represent 9\% of isolated malformations and are found in $16 \%$ of polymalformative syndromes [3]. There are many varieties of brain malformations. The incidence depends on the type of malformation. The most frequent BM is agenesis of the corpus callosum. Dandy Walker malformation (DWM) is a genetically and sporadic disorder of cerebellar development with an estimated incidence of 1/30,000 live births [4] while the incidence of Sturge Weber syndrome is currently about 1/500,000 live births, hemimegalencephaly is much rare and its incidence is unknown [5]. BMs are the major causes of epilepsy, psychomotor development abnormalities and other neurological disorders. The neuroimaging technique of choice for diagnosis of these malformations is magnetic resonance imaging (MRI) [6], but unfortunately MRI is expensive, and is not available in a poor resource country like Cameroon. CT scan associated to clinical signs can help to suspect or to confirm a malformation. The authors report here three cases of malformations discovered during cranial CT scan at the regional hospital Ngaoundere.

\section{Case Report 1}

A two month-old boy was admitted in our hospital because of macrocephaly, diarrhea, vomiting and fever evolving since one week. As first care, oral salt rehydration has been managed. The interrogation revealed that the delivery has been made at home after an unfollowed pregnancy and vaccines were adminstered in retrospect at the refugee's camp. Physical examination on admission revealed a very irritable child with a general lethargic state. The head circumference measured $50 \mathrm{~cm}$ with the shape of the head in dome (Figure 1) and the skin of the skull was thinned with venous dilatation; the sticking out and tense open fontanelles. The eyes had an aspect in sunset. The neurological exam revealed a low tone (hypotonia). The examination of other systems revealed signs of dehydrations (dry lips, persistent cutaneous folds, absence of tears). Routine investigations were essentially normal, but Full Blood Count revealed a light anemia. Brain CT scan revealed a cystic dilatation of the fourth, third and lateral ventricles which enlarged posterior fossa and agenesis of the corpus callosum and the cerebellar vermis (Figure 2). Given the history and clinicoradiological features, the diagnosis of Dandy Walker was made and the child was referred to neuro surgery service in yaoundé and he died two weeks ago.

\section{Case Report 2}

A two days old boy had been admitted for consultation for bursts of flexion spasm (start-up type). The interview revealed no history of fetal distress, pregnancy and delivery were normal, the Apgar score was 9/10 and the birth weight was $3500 \mathrm{~g}$. The child presented at birth a large depigmentation-like spot (Figure 3 ) covering the frontal region and the corner of the right eye (facial angioma 
plane of wine) for which his parents consulted and the vitiligo diagnosis was made. Psychomotor development was normal until the onset of epileptic seizures.

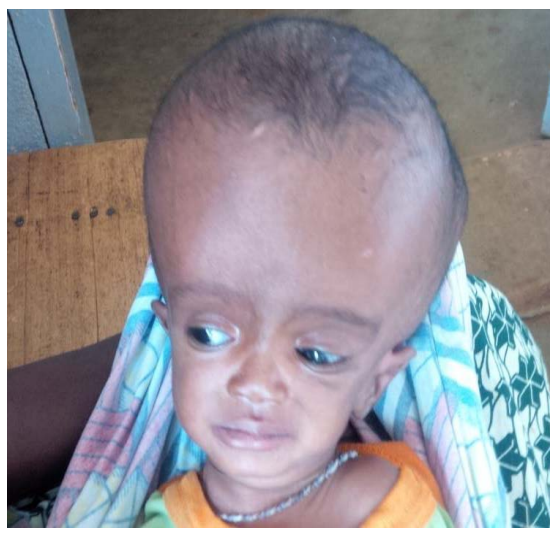

Figure 1. Two month-old boy with macrocephaly.

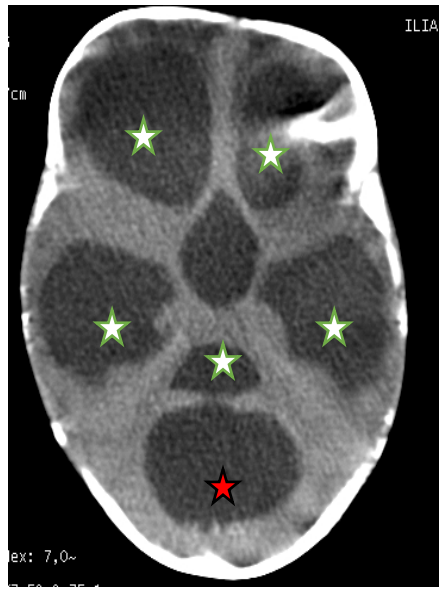

(a)

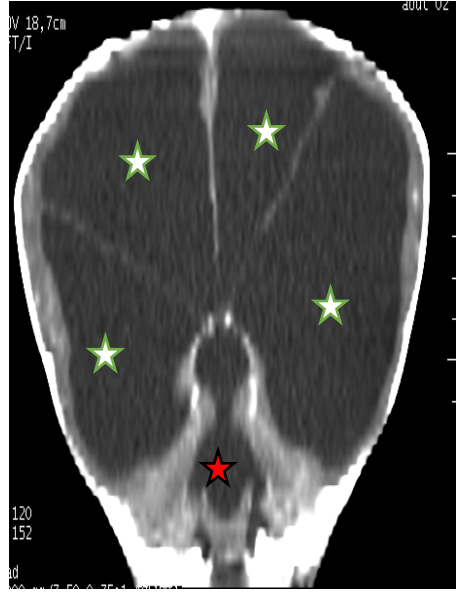

(b)

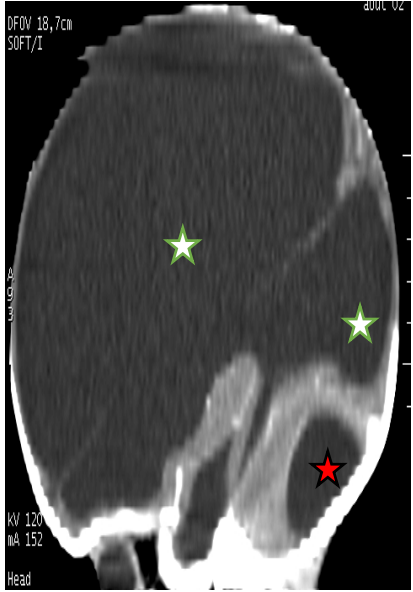

(c)

Figure 2. An axial (a), frontal (b) and sagital (c) computed tomography (CT) scan showing a large dilatation of ventricular system (white stars) and a cerebrospinal fluid cyst (red star) in the posterior fossa. 


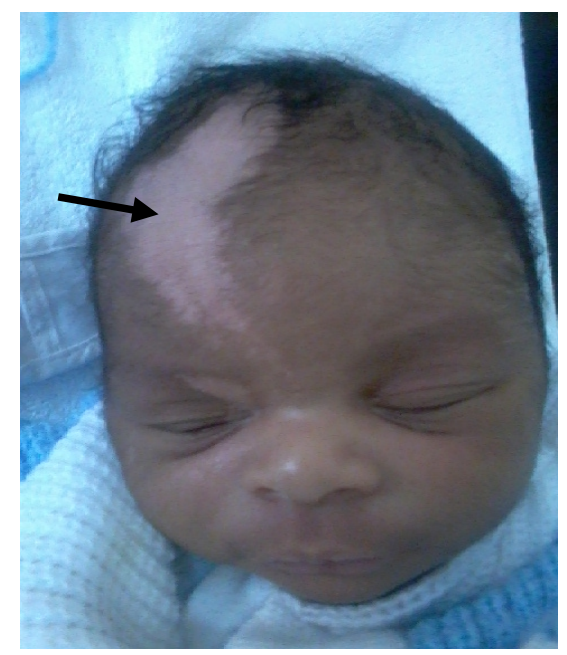

Figure 3. Port wine stain interresting Frontal branch of V1 (arrow).

The cranio-encephalic CT performed in a $2.5 \mathrm{~mm}$ contiguous helix, without injection of contrast agent, showed gyriform calcifications, showing posterior right parietal cerebral convolutions associated with sub-cortical and cortical atrophy and enlargement of the ventricular system and tanks from the base of the skull (Figure 4). Faced with these very evocative images of Sturge Weber's disease, he was referred to Yaoundé in a neuropediatric ward for good care. We did not have information regarding its evolution.

\section{Case Report 3}

A four month old boy was admitted to a pediatric consultation for epileptic seizures. The interrogation found a notion of recurrent convulsions since the age of 4 weeks. At the age of one month, the parents noticed a reduction in the mobility of the right hemi-body. Pregnancy and delivery were normal. Physical examination revealed cranial asymmetry, increased head circumference, sutural separation, and prominent fontanels. The neurological examination revealed a left hemi-corporeal motor deficit with spasm and left facial palsy, suggesting an epileptic state on cerebral malformation. A CT scan was performed demonstrating cerebral hemisphere asymmetry with hypertrophy of the right hemisphere with deviation of midline structures to the left, thickening of the right frontal cerebral cortex, and moderate enlargement of the right lateral ventricle consistent with hemimegalencephaly (see Figure 5). The child was also referred to neuropediatric service in Yaoundé and he died a month ago.

\section{Discussion}

Most central nervous system malformations begin long before a baby is born. The human brain is a complex organ and its development involves many interactions. Several events are responsible for the formation of a mature brain such as: neurulation, neurogenesis, migration of neural crest cells, neurotransmitter 


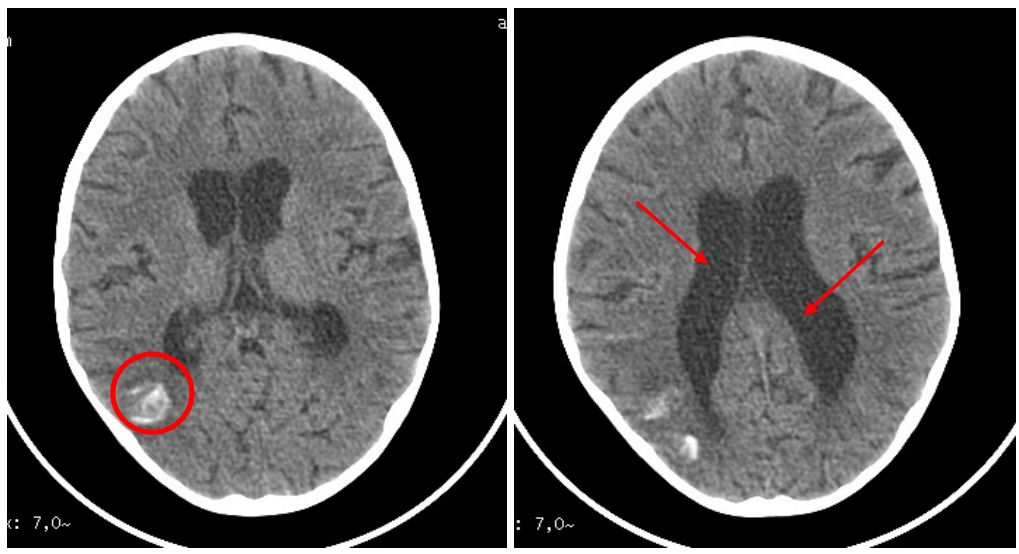

Figure 4. Axial cut at the level of the lateral ventricles computed tomography without contrast enhancement showing gyriform calcifications (red circle), with enlargement of lateral ventricles (red arrow).

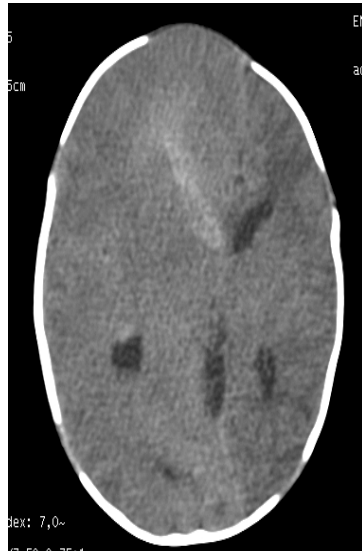

(a)

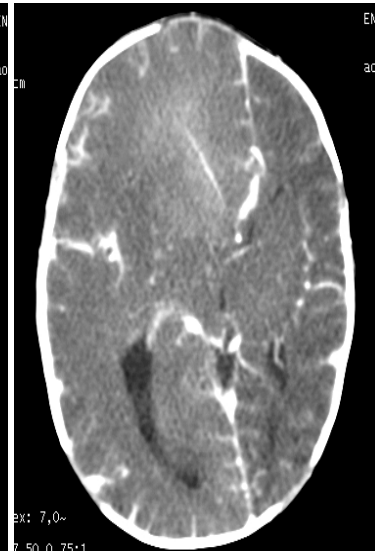

(b)

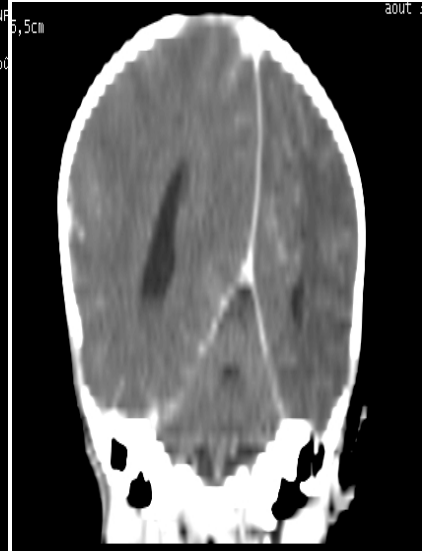

(c)

Figure 5. Non contrast (a) and contrast axial (b) and frontal (c) CT images of the brain in a four-month-old boy with hemimegalencephaly and diminished volume of the controlateral hemisphere. The right cerebral hemisphere is hypertrophic and agyric, and the right lateral ventricle is enlarged on the axial (a) and frontal contrast view (c).

biosynthesis and myelination. Alteration of only one of these processes, at the molecular or cellular level, can lead to the appearance of a wide variety of malformations. Most birth defects happen during the first 3 months [7]. Dandy-Walker malformation (DWM) is a rare group of congenital human brain malformations. It was first described by Dandy and Blackfan in 1914 [8]. Signs and symptoms are caused by abnormal brain development present at birth or develop within the first year of life. DWM is a genetically and sporadic disorder of cerebellar development with an estimated incidence of 1/30,000 live births, which occurs more frequently in females than in males, the age at diagnosis varies from birth to older childhood [4] which remains coherent with our case. In most patients, the cause of Dandy-Walker malformation is not known, a few cases resulting from autosomal recessive genes have been reported. Predisposing factors include gestational (First trimester) exposure to rubella, cytomegalovirus, 
toxoplasmosis, warfarin, alcohol, and isotretinoin [9]. Its results from defects in the development of the roof of the fetal rhombencephalon along with a partial or complete lack of patency of the foramen of Magendie. It is known as the most common posterior fossa malformation [10]. Classically, posterior fossa cystic malformations have been divided into Dandy-Walker malformation, Dandy Walker variant, mega cisterna magna, and posterior fossa arachnoid cyst [9] [11]. Medical literature identified four classic signs of this malformation: complete or partial agenesis of the vermis of the cerebellum; cystic enlargement of the fourth ventricle; enlargement of the posterior fossa; and cranial displacement of the tentorium cerebelli with anti-clockwise rotation of the vermis. DWM is sometimes $(20 \%-80 \%)$ associated with hydrocephalus like in our case, in which blocage of the normal flow of the cerebrospinal fluid leads to excessive amount of fluids in and around the brain. Sturge Weber syndrome (SWS) is a neurocutaneous disorder that is associated with facial capillary malformation, Port wine stain (PWS), glaucoma, and leptomeningeal angioma in its complete form. Its incidence is estimated to be $1 / 20,000$ - 50,000 live births [12]. Many authors hypothesised that the cause of SWS might be a somatic mutation, and this would explain its sporadic appearance. PWS is the characteristic cutaneous manifestation in SWS which is present at birth, of variable size, normally on one side though it may be bilateral, and of a color ranging from pale pink to purple. To date, studies have stratified risk assuming that facial PWS are distributed according to the areas of sensory innervation of the trigeminal nerve branches: the frontal branch (V1), the maxillary branch (V2), and the mandibular branch (V3). All studies agree that lesions with a highest risk of involvement are those affecting the V1 branch, the upper eyelid, or those that are bilateral and extensive [13]. In our case the child presented at birth a large depigmentation-like spot covering the frontal region and the corner of the right eye.

Ophtalmologic involvement which is present in 30\% - 70\% of SWS [14] were not found in our case. There are 3 types of Sturge-Weber Syndrome: type I is the classic associating a plane angioma of the face and leptomeningeal angioma, type II with presence of only facial angioma and type III with presence of only leptomeningeal angioma. Type III is the rarest and only a few cases have been described in the littérature [15], our case was type I. Hemimegalencephaly or unilateral megalencephaly is a rare congenital disorder of cortical development in which there is hamartomatous overgrowth of all or part of a cerebral hemisphere resulting in cerebral asymmetry. The affected hemisphere may show agyria, pachygyria, polymicrogyria or hamartomatous malformation while the opposite hemisphere remaind usually normal [16]. Hemimegalencephaly is characterized by enlargement of whole or part of one brain hemisphere. Although the mechanism of hemimegalencephaly is not fully understood, it is thought to result from an insult to the developing hemisphere during the second trimester of pregnancy due to abnormal neuronal migration in the third week of gestation due to the disorder of genetically programmed development linked to cellular 
lineage and establishment of symmetry. Other proposed causes for hemimegalencephaly have included a biochemical defect in control of cell metabolism, and an abnormal number of chromosomes. It can occur isolated or in association with neurocutaneous syndromes or developmental disorders like KlippelTrenaunay-Weber syndrome, It's hypomelanosis, neurofibromatosis, and tuberous sclerosis complex. [17]. In our observation the hemimegalencephaly was isolated. It is a very rare anomaly. To the best of our knowledge the frequency of these malformations is not known in sub-Saharian Africa. Some cases has been reported by some authors which can be isolated or either associated to controlateral hemispheral volume loss or either to tuberosis sclerosis [16] [17].

Plain X-ray is not a technique of choice, it is possible to observe the classical gyriform cortical calcifications also known as railroad track appearance, which affect the intimal layer of the meningeal arteries in SSW [13]. The neuroimaging technique of choice for diagnosis is gadolinium contrast magnetic resonance imaging, since it can reveal leptomeningeal angiomatosis and the extent to which it affects brain structures in SSW [18]. Even if plain radiographs of the skull may show some characteristic features of DWM, such as enlargement and deepening of the posterior fossa associated with elevation of the groove for the transverse sinus and torcular, at the present time CT scan and MRI are the most useful methods of diagnostic imaging. CT scan easily demonstrate a midline defect of the cerebellum which involves the caudal vermis. MRI has the advantage of multiplanar imaging and better anatomic resolution and should be considered as the gold standard to investigate posterior fossa cystic malformations [19].

MRI can best define the relationship between the cyst and the fourth ventricle and can detect vermian rotation and the presence of signs of vermian dysgenesis and allows surgeons to view the cerebellum and associated structures accurately and to determine which form the malformation has taken and to what extent the DWM has progressed [20]. Unfortunately MRI is expensive, it requires patient cooperation or sedation and is not available everywhere in Cameroon. Ultrasound (US) may be the initial examination performed in case of DWM because it can be done portably and without sedation. DWM may be diagnosed in utero by using ultrasound as early as 14 weeks of gestation in infants with open fontanels, hydrocephalus and some posterior fossa abnomalies that can be detected. In expert hands, ultrasound may help in the differentiation between arachnoid cyst, large cistern magna and a real DWM. However US is limited because it is heavily operator-dependent. Abnormalities such as the gyral, dural, tentorial, and skull abnomalies that accompany Dandy-Walker malformations are not clearly detected by US [21]. None of our patients were diagnose in utero, giving evidence of poor pregnancy monitoring.

Brain CT scan is a technique used frequently to assess children who present with hemiparesis or seizure. In DWM, CT scan can distinguish hydrocephalus associated with Dandy-Walker and hydrocephalus with other etiologies, despite the fact that clearly distinguishes subtypes of the Dandy-Walker complex on axi- 
al CT images is difficult and it exposes the infant to ionizing radiation [4]. our case presented with large dilatation of ventricular system and a cerebrospinal fluid cyst in the posterior fossa. The characteristic findings of SWS are presence of reduced parenchymal volume, enlarged ventricle, and enlarged choroid plexus, we only found gyriform calcifications with enlargement of lateral ventricles in our case. From one year of age, computed tomography can detect calcifications, which would not normally be visible in a plain X-ray. Computed tomography is also more sensitive for detecting calcifications in SWS than MRI [13]. Although the diagnosis of hemimegalencephaly can be suspected early on clinical signs, $\mathrm{CT}$ and MRI confirm the diagnosis [22]. As imaging findings in hemimegalencephaly, there is an increase in volume of the hemisphere reached with, within it, an enlarged and dysmorphic ventricular system. The lateral ventricle within the affected hemisphere is both enlarged in size and dysmorphic [6]. The diagnosis of hemimegalencephaly is confirmed by cerebral MRI showing significant asymmetry with hemispheric size increase, dysplastic cortex and asymmetry with ventricular formation. In our case CT presentation was classical with the right cerebral hemisphere which is hypertrophic and agyric, and the right lateral ventricle is enlarged, this was coherent with the others cases described by the other authors [16] [17]. The aspect is variable but some signs are constant and characteristic. The enlargement of at least one lobe is present in all the patients, of variable importance, from discrete to severe. In more than $50 \%$ of cases the entire hemisphere is enlarged [23]. The CT scan is sometimes normal although asymmetry of the skull is obvious; extensive or more localized calcifications in the white or gray matter are sometimes present. MRI is the method of choice and shows an increase in white matter volume of the pathological hemisphere that has signal abnormalities and a maturation advance [21].

\section{Conclusion}

Brain malformation remains a rare case. The clinical manifestations of brain malformations are variable and depend on the type. Their diagnosis is difficult and base on imaging. These cases illustrate that in a poor-country resource like Cameroon, where MRI is not available, computed tomography combined to clinical symptoms can help to diagnose the brain malformations for early referral to better equipped centers for a good care. These cases contribute to the knowledge of this rare case, and emphasize the importance of CT scan on their diagnosis in absence of MRI.

\section{Conflicts of Interest}

The authors declare that they have no conflicts of interest.

\section{References}

[1] Brain Malformations (National Library of Medicine). Health Topics. https://medlineplus.gov/brainmalformations.html

[2] Moifo, B., Azegha Jiotsa, R., Nguefack, S., Tatah, S., Mah, E., Dongmo Nguefack, F. 
and Mbonda, E. (2017) Prevalence and CT-Scan Presentations of Brain Malformations in Children at a University-Affiliated Mother and Child Hospital (Cameroon). Open Journal of Medical Imaging, 7, 220-228. https://doi.org/10.4236/ojmi.2017.74020

[3] Lépinard, C. (2011) Chapter 11-Brain Malformations. In: Bonneau, D., Ed., Prenatal Diagnosis in Practice, Elsevier Masson, Paris, 103-130.

[4] Calleja, M.R.C., Verdú, A. and Félix, V. (2003) Dandy-Walker Malformation in an Infant with Tetrasomy 9p. Brain \& Development, 25, 220-223

http://www.elsevier.com/locate/braindev https://doi.org/10.1016/S0387-7604(02)00211-5

[5] Kelekele, J.K., Kayembe, D.L., Mwanza, J., Tshibassu, P., Lukusa, P.T. and Kakiese, V. Syndrome de Sturge-Weber et de Klippel-Trenaunay chez un garçon de 19 ans à Kinshasa Overlap of Sturge-Weber syndrome and Klippel-Trenaunay syndrome in a 19 years ol boy in Kinshasa.

[6] Jerbi Omezzine, S., Zantour, B., Chouchene, N., Sfar, M.T. and Hamza, H.A. (2013) Hemimegalencephaly: Role of MR Imaging. Archives de Pédiatrie, 20, 418-420.

[7] Graham, J.W. (2007) Smith's Recognizable Patterns of Human Deformation. 3rd Edition, Saunders, Philadelphia, 3.

[8] Dandy, W.E. and Blackfan, K.D. (1914) Internal Hydrocephalus: An Experimental, Clinical and Pathological Study. American Journal of Diseases of Children, 8, 406-482. https://doi.org/10.1001/archpedi.1914.02180010416002

[9] Hamid, H.A. (2007) Dandy-Walker Malformation, Egypt. Journal of Human Genetics, 8.

[10] Bosemani, T., Orman, G., Boltshauser, E., et al. (2015) Congenital Abnormalities of the Posterior Fossa. Radiographics, 35, 200-220.

https://doi.org/10.1148/rg.351140038

[11] Incesu, L. and Khosla, A. (2013) Imaging in Dandy-Walker Syndrome. http://emedicine.medscape.com/article/408059-overview

[12] Sudarsanam, A. and Ardern-Holmes, S. (2013) Sturge-Weber Syndrome: From the Past to Thepresent. EJPN, 18, 258-326.

[13] Higueros, E., Roe, E., Granell, E. and Baselga, E. (2017) Síndrome de Sturge-Weber: revisión. Actas Dermosifiliogr. https://doi.org/10.1016/j.ad.2016.09.022

[14] Wirth, M., Bazard, M.C., Schmitt, E., Rouabah, M. and Hascoe, J.M. (2017) Ophthalmological Disorders in Childhood with Sturge-Weber-Krabbe Syndrome. Pediatric Archives, 24, 36-40. https://www.sciencedirect.com/

[15] Kamoun, F., Sfaihi, L., Hentati, Y., Baklouti, K., Kamoun, T., Ben, K., Mahfoudh, B., Mnifb, Z. and Hachicha, M. (2013) Clinicl Case: Chronic Septic Gralnulomatosis and Sturge Weber Syndrome, a Frotuitous Association? Journal of pediatrics and Child Care, 26, 332-335.

[16] Sener, R.N. (1995) Hemimegalencephaly Associated with Contralateral Hemispheral Volume Loss. Pediatric Radiology, 25, 387-388. https://doi.org/10.1007/BF02021717

[17] Balaji, R., Kesavadas, C., Ramachandran, K., Dinesh Nayak, S. and Priyakumari, T. (2008) Longitudinal CT and MR Appearances of Hemimegalencephaly in a Patient with Tuberous Sclerosis. Child s Nervous System, 24, 397-401. https://doi.org/10.1007/s00381-007-0463-x

[18] Marana Pérez, A.I., Ruiz-Falcó Rojas, M.L., Puertas Martín, V., Domínguez Carral, J., Carreras Sáez, I., Duat Rodríguez, A., et al. (2017) Análisis del síndrome de 
Sturge-Weber: Estudio retrospectivo de múltiples variables asociadas. Neurología, 32, 363-370. https://doi.org/10.1016/j.nrl.2015.12.012

[19] Cakmak, A., Zeyrek, D., Cekin, A. and Karazeybek, H. (2008) Dandy-Walker Syndrome Together with Occipital Encephalocele. Minerva Pediatrica, 60, 465-468.

[20] Reese, V., et al. (1993) Association of Dandy-Walker Malformations Facial Hemangiomas with and Other Posterior Fossa. The Journal of Pediatrics, 122, 379-384.

[21] Bulteau, C., Oliver, M.V. and Fohlen, M. (2008) Hemimegalencephaly: Genetic, Clinical and Histological Aspects. Epilepsy, 20, 274-280.

[22] Cagneaux, M. and Guibaud, L. (2013) Imaging of Supratentorial Malformative Pathologies. Pediatrics, 16, 179-192.

[23] Spennato, P., Mirone, G., Nastro, A., Consiglio Buonocore, M., Ruggiero, C., Trischitta, V., Aliberti, F. and Cinalli, G. (2011) Hydrocephalus in Dandy-Walker Malformation. Childs Nervous System, 27, 1665.

https://doi.org/10.1007/s00381-011-1544-4 